\title{
On the Propositional Attitudes
}

\section{KEITH COLEMAN}

The University of Kansas

An adequate account of the nature of the so called propositional attitudes' has long been sought by philosophers of mind, and most theories which have been proposed have suffered from certain logical or epistemological defects or have been limited in scope. The issues centering around the nature of beliefs, desires, and the other attitudes pertain to two principal areas of investigation: the explication of a (psychological) theory concerning the objects of the propositional attitudes and the analysis of the logical structure of such sentences as those with the form 'A believes that $\mathrm{P}$ ' or 'A wishes (desires) that $\mathrm{p}$.' My main concern in this paper is with problems associated with the latter endeavor. Such problems in an important respect are more basic, for a successful attempt at characterizing the typical form of sentences that express propositional attitudes must be made before any theory about the objects of beliefs, desires, and other intentions can possibly take shape.?

The sentence "Jones believes that there are unicorns" has the typical form of the members of a class of sentences each one consisting of one or more names or denoting phrases followed by an intentional idiom of the kind 'believes that', 'desires that', 'wishes that', 'hopes that', 'fears that', 'says that', 'wonders whether', etc. followed by a complete sentence. Such complex sentences when taken as assertions have usually been seen as expressing a relationship (or a propositional attitude) of one kind or another between an object (usually a person) and a belief, desire, wish, hope, or other intention (or object of the propositional attitude). For the sake of facilitating the discussion regarding these sentences, I shall, with a certain degree of liberty, call such idioms consisting of the word 'that' preceded by a verb of intention 'proposing phrases'; I seek then an analysis of the role such phrases have in determining the logical form of sentences in which they occur. I will begin my account by specifying the logical requirements any such analysis must meet and then briefly examine some of the proposed analyses (each one of which I believe fails to satisfy at least one requirement) and then end by presenting and justifying my analysis, which, hopefully, does meet these requirements.

1 I use the words 'intention' and 'intentional' where I have in mind words or sentence fragments that refer to the propositional attitudes or to the socalled objects of these attitudes. As will be pointed out later, intentional contexts are often intensional (i.e., non-extensional). 
In an interesting, albeit somewhat lengthy, article, Jerry Fodor carefully delineates a scries of conceptual conditions which must be satisfied by any theory dealing with propositional attitudes. Although Fodor is primarily concerned with developing a theory that will eventually mesh with the empirical results of cognitive psychology (the first of the endeavors previously mentioned), many of his observations are relevant to the analysis of the inferential structure of sentences containing proposing phrases. The questions concerning logical form are not always easy to separate from the questions associated with the structure and nature of the empirical or conceptual theories of the propositional attitudes, but in what follows I will try to focus solely on issues pertaining to the former. Many of my remarks in the next few paragraphs have their origins in Fodor's paper.

First of all, the surface grammar of typical sentences of the kind in question would seem to indicate, under a fairly natural reading, that proposing phrases, as was noted above, introduce a two place relation between something that can have (or enter into) a propositional attitude and what has been called the 'object' or the 'content' of the attitude. The embedded sentence of the 'that'-clause then reveals the (propositional) content of the particular attitude. Syntactically, any such sentence can be constructed from a singular term, a proposing phrase, and a sentence. ${ }^{2}$ Where ' $R$ ' represents a proposing phrase and $a$ is any term and $p$ is any sentence, we have $r_{a R p}{ }^{7}$ as the apparent form of such a sentence. The trouble with this rendering of proposing phrases is that it does not accord well with any standard first order language. There simply are no sentence forming operators on names and sentences. ' $R$ ' cannot be a sentential connective, if for no other reason than that $a$ is a term (name or denoting phrase) and not a sentence. In addition, $p$ is a sentence and hence does not denote an object ${ }^{3}$, so that its occurrence in the complex sentence seems out of place. In spite of all this, the inclination to treat ' $R$ ' as a relation is re-enforced when one considers that by interchanging the

2 There are some sentences that do not contain proposing phrases but nevertheless can be regarded as synonymous with at least one sentence that does contain such a phrase. As Quine has pointed out, a sentence such as "I want a sloop" is equivalent to the sentence "I desire that I have a sloop." Similar transformations for other sentences of the same general type would seem to be available.

3 If one were to agree with Frege and take a sentence as referring to its truth value, then a sentence such as "Tom believes that it is raining" would express a relationship between Tom and a truth value, but this obviously is not what this sentence is about. 
positions of $a$ and $p$ the complex, ${ }^{r_{p}} \boldsymbol{R a}{ }^{\top}$, if even meaningful, does not in general have the same sense as $\left.r_{a} R p\right\urcorner$, and yet for every ' $R$ ' there is a related construction ' $R^{*}$ such that $r_{p R^{*} a}{ }^{7}$ is true iff $r_{a} R_{p}{ }^{7}$ is true. For example, if the sentence "Jones believes that unicorns exist" is true, then, despite the fact that "Unicorns exist believes that Jones" is not meaningful, the sentence "That unicorns exist is believed by Jones" is both meaningful and true. This parallels the account that can be given of more common place relations: for instance, if "Susan loves Bill" is true, then "Bill is loved by Susan" is also true. It is difficult, then, on the one hand, to treat a proposing phrase as anything other than a two-place predicate; however, on the other hand, the exact manner in which the term and the embedded sentence are linked by the proposing phrase needs to be clarified. If the linkage cannot be made with the logical apparatus of a first order language, then this would indicate the need for a more enriched language.

The relational aspect of proposing phrases is also apparent in the kind of inferences which can be made from sentences containing such phrases. Consider the following sentence.

(1) Joe believes that Tom is a jerk.

It can be concluded from (1), by existential generalization, that

(2) Joe believes something is true. What this means is that quantification over the position occupied by the 'that'-clause is permissible so that the noun clause is also functioning from a logical perspective as a denoting expression. This again indicates that the proposing phrase (or at least a part of it) is a two-place predicate. The story is further complicated, though, by the fact that from (1) it follows that

(3) Joe believes something about Tom.

If the "that'-clause in (1) denotes the sentence "Tom is a jerk", then the inference from (1) to (3) is reasonable since sentences can be characterized by their subject. The inference is nonetheless unusual since the property of the sentence could be inferred from (1), seemingly without any additional assumptions, just by the form of the 'that'- clause. It's also true from (1) and the sentence,

(4) Mary believes that Tom is not a jerk

that Mary and Joe disagree about Tom. In this case, however, additional assumptions about what constitutes a disagreement are needed. Furthermore, if "Tom is a jerk" is true, then from (1) we can conclude not only (3) but also,

(5) Joe believes something true about Tom.

These last few observations suggest that what is referred to by the 'that'clause is a sentence, a sentence which can immediately take part in subsequent derivations. 
If we take a proposing phrase as introducing a relation between a person and a sentence, 4 then we can understand the similarities between sentences of the form 'A says that $p$ ' and sentences of the form 'A says ' $p$ ", but we must account for both the similarities and the obvious differences between these forms of, respectively, indirect and direct discuurse. Although both of the sentences,

(6) Galileo said, "The earth moves"

(7) Galileo said that the earth moves

appear to be expressing a relation between Galileo and a sentence, 5 neither the truth values (in this case) nor the deductive consequences are quite the same. Since Galileo did not speak English, (6) is, literally, false, but (7) is true. Although (6) can plausibly be said to entail (7), and hence every sentence it entails, $(7)$ and, at least ostensibly, every sentence it entails does not, jointly nor individually, entail (6). The inference, then, from (7) to (6) would be illicit. Also, the sentence "Galileo said something" seems to follow directly from (6) and also from (7). However, it should be noted that the inference from (6) to (7) depends on an assumption about how Galileo's saying should be interpreted. If he were merely joking, for instance, then his saying of (6) would not constitute a legitimate assertion and thereby would not be a 'saying that' (where a 'saying that' is an assertion about the content of what was said in the assertoric mode). There is also another kind of asymmetry between the logical consequences of 'sayings' on the one hand and of 'sayings that' on the other. Consider the following sentences.

(8) George said, "There are unicorns in Georgia."

(9) George said, "In Georgia, there are unicorns."

(10) George said that there are unicorns in Georgia.

(11) George said that in Georgia there are unicorns.

4 The manner in which we understand this relationship as obtaining does not really matter in the discussion to follow. There are perhaps many ways a psychological theory could in principle provide content to this relation. My only point here is that the logical structure of sentences containing proposing phrases seems to require that we take such sentences as asserting some kind of relationship between an object and a sentence. A psychological theory may even end up positing entities that are said to be the objects of the propositional attitudes or may even correlate, or identify, the propositional attitudes with certain definite mental or physical states.

5 The relations referred to in the two sentences cannot be the same. The relation that will obtain if (6) is true holds between Galileo and one particular sentence, whereas the relation that will obtain if $(7)$ is true holds between Galileo and any sentence synonymous with the particular sentence referred to in (6). Beyond this, how we construe these relations does not really matter. See note 3 . 
Sentences (10) and (11) entail one another, while sentences (8) and (9) do not. (10) and (11) may indeed be considered synonymous, while (8) and (9) appear similar but not synonymous. These observations suggest that there are logical and non-logical relations between types of sentences exemplified by (6) and (7), and, hence, any account of the logical nature of such sentences must render these relations explicit.

Perhaps the most celebrated feature of proposing phrases is that in many cases they set up intentional contexts into which quantification cannot proceed and within which substitutions of co-referring terms will not in general preserve truth values. As an example of the former, if Joe fancies that only the present king of France is bald, then the sentence,

(12) Joe believes that the present king of France is bald is true, whereas the sentence, in partial symbolic dress,

(13) $(\exists x)$ (Joe believes that $x$ is bald)

is false. Hence, (12) cannot be said to entail (13). As an example of the failure of the substitutivity principle, consider the following pair of sentences.

(14) Joe believes that Mark Twain wrote Huckleberry Finn.

(15) Joe believes that Samuel Clemens wrote Huckleberry Finn.

Even though Mark Twain is Samuel Clemens, (14) may very well be true without (15) being true. Joe, for instance, may have an extensive knowledge of American literature but not know who Samuel Clemens was.

Our translations into canonical notation of sentences expressive of propositional attitudes must explain why such intentional contexts are referentially opaque. Although this opacity has traditionally been regarded as one of the distinguishing features of these types of sentences and is the main reason for their logical intractability, the intentional contexts that proposing phrases introduce are not always completely opaque. For instance, (14) could be said to entail (15) if the sentence,

(16) Joe believes that Mark Twain is Samuel Clemens

were true and we made certain rationality assumptions about the completeness and coherency of Joe's belief system. More specifically, if Joe's belief set were closed under the logical consequence relation, i.e. if Joe believes whatever is entailed by any combination of Joe's other beliefs, then (14) and (16) would jointly entail (15). Also, if we were to regard, for some reason or other, the sentences following the 'that'-clauses in (14) and (15) as synonymous, (14) and (15) would both be true. or both be false However it is, then, that we finally analyze the logical nature of intensional contexts must account for the degree of, and not the mere fact of, the opacity which they introduce.

Quine has contended that at least for the propositional attitudes of believing, desiring, and striving there are two different modes in which one 
could be said to have such an attitude. 6 These correspond to the two different senses attached to a sentence such as,

(17) Ralph believes that there are spies.

If Ralph's believing is in the notional sense, then (17) can be paraphrased as,

(18) Ralph believes that there is someone such that that person is a spy.

On the other hand, if Ralph's belief is understood in the relational sense, then (17) would be paraphrased as,

(19) There is someone of whom Ralph believes to be a spy.

The important difference between (18) and (19) which would affect the way in which sentence (17) would be translated into a canonical notation concerns the extent to which it is possible to quantify into the 'that'-clause in (17). The 'believes that'-clause of (18) can be understood as referring to a dyadic relation between a person and a sentence (or proposition) held true, whereas the corresponding 'believes that'-clause in (19) can be understood as referring to a triadic relation between two people and a property. Quine thus renders (18) and (19) as, respectively,

(20) Ralph believes that [there is someone who is a spy]

(21) $(\exists x)$ (Ralph believes $y(y$ is a spy) of $x)$.

Quine later, in an attempt to eliminate reference to properties (such as $y(y$ is a spy): the property of being a spy), suggests that even (21) could be understood as asserting that a relation holds between two objects and a sentence. In this case, Ralph could be said to believe that a sentence is satisfied by some object. In Word and Object, Quine then dispenses with even the reference to sentences by fusing the proposing phrase in a sentence like (20) to the embedded sentence and the intensional verb in a sentence like (21) to the predicate abstraction to form in both cases complex predicate expressions. Sentence (20) would be rendered into canonical notation using a one-place predicate, and (21) would be translated by using a dyadic predicate. In simplified form, (20) and (21) would appear as,

(22) $\mathrm{B}_{1} \mathrm{a}$

(23) $(\exists x) B_{2} a x$

David Kaplan has pointed out a difficulty with Quine's analysis of believing in the relational sense.7 Kaplan charged Quine with being inconsistent when he failed to rule out constructions like (21) and (23) in regard to intentional belief contexts but did rule out similar constructions in regard to non-extensional modal contexts. In order to see what is wrong with these symbolic versions of the relational sense of (17), consider the following sentence.

(24) Holmes believes that someone is the murderer.

6 This is done in "Quantifiers and Propositional Attitudes".

7 See Kaplan's essay, "Quantifying In". 
If the believing mentioned in this sentence is taken to be in the relational sense, then (24) can be rendered according to Quine as,

(25) $(\exists x)$ (Holmes believes $y(y$ is the murderer) of $x$ ).

However, the wrong idea of the relational sense of (24) is given by (25). If

Holmes were only to believe, for instance, that among the several guests at the hotel one of them was the murderer, and hence believed that the hotel guest who committed the murder is the murderer, and if the murderer, whoever that should be, was in fact a hotel guest, then (25) would be true, whereas the relational sense of (24) is that there is some particular individual of whom it can be said that Holmes believes him or her to be the murderer. ${ }^{8}$ In other words, as long as Holmes is familiar with the

8 If Holmes either believed that the hotel guest who committed the murder is the murderer or believed that the murder is the murderer, then (25) would be true on the assumption that there is a unique individual who committed the murder and that individual is also a hotel guest. If the definite description in both cases is treated as a name, then we would have, respectively, where 'the hotel guest who committed the murder' is ' $h$ ' and 'the murderer' is ' $m$ ',

(I) Holmes believes that $h=m$

or

(II) Holmes believes that $\mathbf{m}=\mathbf{m}$.

Since both ' $h$ ' and ' $m$ ' denote some one individual, either one of these sentences entails the sentence, in Quine's notation,

(III) $(\exists x)($ Holmes believes $y(y=m)$ of $x)$,

so (25) is entailed by either one of them. If we were to analyze the definite description in the traditional Russellian fashion, we would obtain, instead of (I),

(IV) Holmes believes that $(\exists \mathrm{z})(\exists \mathrm{x})((\mathrm{z}$ is a hotel guest \& $\mathrm{z}$ committed the murder $)$ \& $(\forall w)((w$ is a hotel guest \& $w$ committed the murder $) \rightarrow z=w))$ $\&((x$ committed the murder \& $(\forall u)(u$ committed the murder $\rightarrow x=u))$ \& $z$ $=x)$ ),

and, instead of (II),

(V) Holmes believes that $(\exists z)((z$ committed the murder \& $(\forall w)(w$ committed the murder $\rightarrow z=w)$ ) \& $z=z$ ).

Now, although this is certainly less obvious than the previous derivation, since there is some one individual who committed the murder (and is also a hotel guest) and Holmes believes this, from (IV) it follows that Holmes believes of someone that that individual both is the hotel guest who is the murderer and is the murderer, so we obtain

(VI) $(\exists x)($ Holmes believes that $y(((y$ is a hotel guest \& $y$ committed the murder $)$ \& $(\forall w)((w$ is a hotel guest \& $w$ committed the murder $) \rightarrow y=w))$ \& (y committed the murder \& $(\forall u)(u$ committed the murder $\rightarrow y=u)$ )) of $x$ ). 
murderer under some description, regardless of how vague, and there is someone who satisfies that description, (25) is true. In contrast, if (24), understood relationally, were true, then the mystery would be solved and the arrest could be made; this meaning is not what is suggested by (25).

In order to fully state the relational sense of (24), Kaplan introduces the notion of a 'vivid name': a name is vivid for a person, roughly, iff, on the assumptions that the name denotes because it is appropriately linked causally with what it denotes and that the name adequately picks out or describes its referent, then it follows that the person knows exactly what or whom it denotes. The idea here is that in order for it to be truly said that Holmes believes of some particular individual that he is the murderer Holmes must be sufficiently acquainted with the murderer such that he knows precisely who the murderer is. Only under these conditions would (24) in the relational sense be true. If Holmes were acquainted with the murderer only by knowing that he can be referred to by some description, say, 'the hotel guest who is taller than five feet and who committed the murder, then (25), but not (24) would be true. As Holmes accumulated more evidence, if a time was reached at which Holmes was acquainted with the murderer by having such a detailed description of him that Holmes could, say, pick him out of a line up, then that description would be sufficiently vivid for Holmes so that he could be said to believe of someone that he is the murderer and (24), understood in the relational sense, would be true. Instead of (25), the symbolic version of (24) in Kaplan's notation is,

(26) $(\exists x)(\exists a)\left(R\left(a, x\right.\right.$, Holmes) \& Holmes $B r_{a}=$ the murderer $\left.{ }^{7}\right)$, where quantification is over objects and names and ' $R(a, x, H o l m e s)$ ' means 'a represents $x$ to Holmes' and 'Holmes $B r_{a}=$ the murderer ${ }^{7}$. means that Holmes believes that the referent of ' $a$ ' and the murderer are the same. The expression ${ }^{\prime} r_{a}=$ the murderer ${ }^{7}$ ' is a complex name that refers to the expression ' $a=$ the murderer' in terms of its syntax. The predicates ' $R$ ' and ' $B$ ' refer to relations between an expression and one or more objects, and ' $R$ ' is defined in such a fashion that any value of ' $a$ ', which will be an expression, that satisfies the propositional function ' $R(a, x$,

Likewise, from (V) we obtain

(VII) $(\exists x)($ Holmes believes that $y(y$ committed the murder \& $(\forall w)(w$ committed the murder $\rightarrow y=w$ )) of $x$ ).

We can then rewrite (VI) as

(VIII) $(\exists x)($ Holmes believes that $y(y$ is the hotel guest who committed the murder \& $y$ is the murderer) of $x$ )

and (VII) as

(IX) $(\exists x)($ Holmes believes that $y(y$ is the murderer) of $x)$, which is (25). Presumably, although I find this a specious inference, (VIII) also entails (25). 
Holmes)' will be a vivid name of $x$ for Holmes. Kaplan argued that such a complicated apparatus was needed in order to correctly render the relational sense of belief.

It should perhaps be noted that in this regard Quine's handling of the distinction between the notional and relational senses of belief is very similar to Bertrand Russell's treatment ${ }^{9}$ of the difference between, respectively, the secondary occurrence of a definite description and the primary occurrence of the description in such a sentence as,

(27) George IV wished to know whether Scott was the author of Waverly.

Although Kaplan is concerned with making clear the relational sense of believing while Russell is concerned with rejecting an analogous sense of wishing to know, Kaplan's observation that Quine has failed to capture in his notation the essential features of this distinction in the case of the two senses of belief parallels the observation that Russell was unable to specify the precise nature of George IV's wonderment in his two paraphrases of (27). If the definite description is given a primary occurrence (i.e., a wide scope), then (27) is rendered as

(28) $(\exists x)((x$ wrote Waverly \& $(\forall y)(y$ wrote Waverly $\rightarrow x=y))$ \& George IV wished to know whether $x=$ Scott).

If the definite description is given a secondary occurrence (i.e., a narrow scope), then (27) is rendered as

(29) George IV wished to know whether $(\exists x)((x$ wrote Waverly \& $(\forall y)(y$ wrote Waverly $\rightarrow x=y$ )) \& $x=$ Scott).

The quantification in (28) is into an intentional context so that this symbolic version of (27) expresses what may be called the relational sense of wishing to know, whereas (29) expresses the notional sense of wishing to know. There are obvious similarities between (28) and (21) on the one hand and between (29) and (20) on the other. If George IV were wanting to know about the law of identity (that is, whether Scott is Scott) and the author of Waverly were Scott, then (28) would be true. Russell came to reject (28) as giving the probable meaning of (27) since (28) would also be true in the case where the author of Waverly was Scott and George IV had seen Scott faintly in the distance and wished only to know whether the person seen faintly in the distance was Scott. Both of these difficulties with (28) are analogous to the difficulties Kaplan claimed made Quine's rendering of (17) as (21) unacceptable.

The trouble with (29) is less apparent, but there is still a problem. Unfortunately, the embedded sentence in (29) also does not represent the exact extent of George IV's wonderment. ${ }^{10}$ Notwithstanding the differences between the primary and the secondary occurrence of the

91 am, of course, referring to Russell's early paper, "On Denoting".

10 On this point, I owe a debt of gratitude to Donald Brownstein, who originally suggested to me that Russell's rendition of (27) with a secondary occurrence of the definite description was incorrect. 
definite description, (27), again depending upon how it is interpreted, would be true in two further distinct cases in only one of which would (29) be true. The first case involves poor George IV pondering the question of the origin of Waverly and wishing to know whether Scott was solely responsible for it in virtue of being Waverly's sole author. The second case involves a more attentive George IV either knowing or taking it for granted that there is one and only one person who wrote Waverly but wishing to know whether or not Scott is that author. The first case corresponds with what is expressed by (29), but the more likely meaning of (27) is such that only if the conditions of the second case were to obtain would (27) be true. The trouble with (29), then, is that too much of the meaning of the definite description is brought into the intensional context; the trouble with (28), on the other hand, is that too little of the meaning of the definite description is brought into the intensional context.

However, even neglecting the difficulties involved in making sense out of the notional versus the relational senses of belief, there is a more telling reason for not accepting Quine's account. By bracketing off, or by fusing, the embedded sentence or predicate expression, respectively, to the proposing phrase or verb in order to form a complicated predicate, one blocks most of the deductive consequences of the types of sentences we have been considering. For example, the inferences from (1) to (2), from (1) to (3), and from (10) to (11) would all be illicit. The only way these inferences could be permitted as sound is through the addition of an indefinite number of non-logical axioms, or perhaps a finite number of axiom schema, which would, problematically, have to be stated in terms of the internal structure of the complex predicate. This, of course, among other things is counter-intuitive, for it would appear that the inferences mentioned above would need no such non-logical support. Furthermore, since the fused predicate expressions would have no logically significant internal structure and would therefore have to be looked upon as (logically) simple monadic or dyadic predicates, this move would be unwarranted since the inferences they support must turn on this internal structure.11

Donald Davidson took a different approach to the problem.12 A sentence such as (7) consists of two sentences juxtaposed; the first sentence mentions what was said by using the demonstrative 'that' to refer to the second sentence, which appears after the 'that' merely to provide the content to what is referred to by the 'that'. The entire construction reports the content of what someone has said, regardless of how they said it or in what language it was said. This is so because the utterance of the

11 However, with regard to its syntax, such a fused predicate expression is nonetheless grammatically complex. See Quine's discussion in Chapter 2 of Philosophy of Logic.

12 See Davidson's essays, "Thought and Talk" and "On Saying That". 
words following the word 'that' is understood to be an utterance of a sentence in the reporter's language synonymous with the sentence in the subject's language which the subject's utterance (which represents a saying of the same thing said by the reporter) is an utterance of. In other words, a sentence like ( 7 ) is true iff there is some utterance that was, in this case, made by Galileo such that Galileo's saying and the reporter's current saying (i.e., the utterance of "the earth moves") make Galileo and the reporter samesayers. For Davidson, any theory giving an account of the nature of sentences expressing propositional attitudes must treat these sentences, and any of the other sentences of the language in which they occur, in a fashion that will allow truth to be defined in a Tarski-like manner. ${ }^{13}$ That is, the truth conditions of any sentence of the language must be recursively specifiable in terms of the truth conditions of the subsentential parts and must take into account the role played by all truth affecting iterative devices in the language. The trouble with the fusion theory of the propositional attitudes is that it does not satisfy this criterion. Since any sentence may follow the proposing phrase in a complex sentence containing one, the strategy of fusing the proposing phrase with the embedded sentence would result in an infinite number of logically simple predicates, and it is hard to see how, on this account, the truth conditions of sentences containing proposing phrases could be characterizable in terms of the truth conditions of their component parts. Learning a fused predicate language would also present difficulties: in order to learn the meaning of a potential infinity of complex sentences containing such predicate expressions, one would have to learn in each case how the meaning of the complex sentence is determined by the meanings of both the proposing phrase (since that construction determines which propositional attitude is being expressed) and the embedded sentence (since that sentence determines the 'content' of the propositional attitude). On Davidson's view, the meaning of any sentence is only specifiable, ultimately, in terms of its truth conditions, but the meaning of the complex sentence could not be given in terms that include, among other things, the truth conditions of the embedded sentence since the syntactically complex predicate is nonetheless logically simple in virtue of its inferential structure.

Davidson's thesis does account for the referential opacity of the embedded sentence in the 'that'-clause; the fact that on the fusion theory this opacity is readily apparent is probably the only good reason for accepting it. Since the meaning of a sentence, or, more to the point, the relations of synonymy, may change upon any type of substitution, the meaning of the sentence following the proposing phrase may not be preserved upon the substitution of co-referring terms. It is this component

13 See Tarski's essay, "The Semantic Conception of Truth and the Foundations of Semantics", or Chapter 3 of Quine's Philosophy of Logic. 
sentence, of course, which determines what the complex sentence is ultimately to be understood as asserting; therefore, substitutions into the former may affect what the latter is about, or its informational content, in that the referent of 'that', i.e., the utterance of the embedded sentence, may change. Also, since the sentence the utterance of which is referred to by 'that' is semantically independent of the sentence consisting of the proposing phrase and everything before it, the truth or falsity of the former sentence has no bearing on the truth or falsity of the latter sentence. Therefore, when one says that something, one is not asserting the something but merely reporting on it; the something may be true or false quite independently of the veracity of the report. This is, of course, the way it should be.

Although Davidson's proposal is attractive in many ways and it allows him to circumvent some serious logical/conceptual problems, it too does not satisfy all the requirements an adequate account must meet. On Davidson's own admission, the sentence in the 'that'-clause is semantically inert and merely occurs in this context so that the utterance of the words following 'that' provides a referent for the demonstrative, so it is hard to see how inferences could be drawn from the content of the syntactically embedded sentence. Also, since sayings that, according to this view, are reports on utterances (in the case of the spoken word) or inscriptions, presumably, (in the case of the written word), 14 just how are we to understand the relation of synonymy between the reporter's words and the subject's word's, for only sentences, not objects, have meaning. Only sentences, and not utterances construed as objects, have truth values. It appears that we again need non-logical axioms (this time, stated in terms of relations between utterances and sentences) in order for us to be able to derive the logical consequences from what was said.

What is even more at the heart of the matter here is that on Davidson's account the meaning of the component sentence is only relevant in determining, in an indirect fashion, the actual truth value of particular complex sentences but is not pertinent to the meaning of the complex sentence. For Davidson, as far as the logical structure of the complex sentence is concerned, the complex sentence stops with the word 'that.' This demonstrative functions as a name so that its reference is needed in particular cases to determine whether a (syntactically, not logically) complex sentence is true or false. The semantics of the complex sentence, though, cannot be separable in this manner from the semantics of the embedded sentence, for one cannot be said to know the meaning of a complex sentence without knowing the meaning of its embedded

14 The fact that 'that' may refer to spoken or written words within a sentence which is either written or spoken means either that the meaning of a 'says that' construction is partially determined by context or that we need a broad interpretation of the word 'utterance'. 
sentence. The situation is different in the case of simple sentences containing names, where knowledge of the referent of the name is not required for knowledge of the sense, or meaning, of the sentence but is required in order to grasp fully the informational content of the sentence and to assess its truth value. Furthermore, since a complex sentence containing the proposing phrase 'said that' is about the same-saying relationship, which involves the relation of synonymy among sentences, and makes reference to a sentence, indirectly by making direct reference to an utterance which in turn is related by an appropriate theory to a sentence, the relationship between the embedded sentence and the remaining portion of the complex sentence must be the relation of an object language to a metalanguage. If the traditional relation between an object language and an associated metalanguage is upheld, then the truth conditions of the component sentence are not part of the truth conditions of the complex sentence since a specification of the truth conditions of the latter sentence will involve only a name of, and not the content of, an utterance of the former sentence. This means that the truth conditions, and hence meaning, of the complex sentence cannot be stated in terms of the truth conditions, and hence meaning, of all of its relevant parts so that Davidson's own account also fails to meet the standard concerning the amenability to a Tarski-like truth definition which Davidson himself explicitly acknowledges must be satisficd by any such theory.

Davidson's theory dealt only with sentences of the form of (7), (10), and (11); it is unclear whether or not his account can be extended to cover sentences expressive of the other propositional attitudes. An account of believing that parallel to the account of saying that would construe the referent of 'that' in a sentence containing the proposing phrase 'believes that' as the utterance of the words following 'that', and the sentence containing the proposing phrase would be true just in case an utterance of the person reported on is such that the current utterance of the words following the word 'that' in the 'that'- clause of the reporter make reporter (if he were to have the same belief as the subject) and subject 'samebelievers'. However, consider the following conversation between two people who up to this time have never said anything disparaging about one another.

Joe says (to Tom), "You are a jerk."

Tom says (to Joe), "Yes, 1 have always believed that you are a jerk." Tom's utterance of the words "You are a jerk" are not the words he should have to utter if he and Joe shared the same belief, even though Joe also utters these words, if for no other reason than that the word 'you' in the mouths of the two speakers does not refer to the same person. In addition, Tom's current utterance of the words "You are a jerk" cannot, by hypothesis, be associated with a past utterance of Tom in virtue of which he now asserts that he believes the same thing as he did in the past. 
Now, consider a modification of the above case with the conversation proceuting instead as follows.

Joe says (to Tom): "You are a jerk."

Tom says (to Joe): "I believe that."

Presumably, Joe and Tom are in agreement here. But to what utterance does Tom's utterance of the word 'that' refer? It cannot refer, again by hypothesis, to any remark that Tom has made in the past. If it should refer to Joe's utterance of "You are a jerk", then it would appear that Tom has, in effect, used Joe's utterance to make a personal comment about Joe, but this does not seem to be what has transpired in this conversation. On the contrary, Tom appears to be admitting here that he believes what Joe says about him, and what Joe says about him is that Tom is a jerk. Thus, Joe's utterance, once the antecedent of the pronoun 'you' is understood in the context of Joe's utterance, can be construed as an utterance of the sentence "Tom is a jerk", and, as a result, the utterance of 'that' in the context of Tom's utterance can be scen as a reference to this sentence. In other words, the word 'that' in the mouth of Tom does not refer to the utterance of Joe but refers to the sentence of which Joe's utterance is, in effect, an utterance of.

A consideration of a host of similar cases reveals the inadequacy of a 'Davidsonian' type of approach to analyzing sentences of the form 'A believes that $p^{\prime} .15$ Davidson's account of 'saying that', even if it were successful, is limited in scope and evidently does not provide us with the key to understanding the nature of the rest of the proposing phrases. We desire to have an account of all the propositional attitudes and hence should prefer a theory that gives a complete account over one that merely gives a partial analysis.

The positive and negative arguments thus far presented support a view which I now wish to advocate. Firstly, at least some sentences containing proposing phrases represent one type of sentence which has a logical structure best understood as relating an object and a sentence. Since such sentences contain syntactically embedded sentences which are nevertheless semantically significant, we must reject any view that always regards the occurrence of the embedded sentence as an orthographic

15 Of course, simpler examples could have been given here to indicate the trouble with such an account of 'believing that'. If the 'believing that' relation were analogous to Davidson's 'saying that' relation, then it would mean that we could only attribute a belicf to a person who had in fact made an appropriate utterance. I chose the above examples both to illustrate the difficulties with this approach and to suggest, at least in part, what the true nature of this relation is. 
accident', as Quine puts it, or as an occupier of a logical lacuna. Both Quine's account and Davidson's account must be discounted on this score. Davidson considered the grammar of sentences containing proposing phrases as misleading. 'That'-clauses, instend of referring in one way or another to sentences as they appear to do (since, for one reason, they are noun clauses and seem to name or refer to their embedded sentences or to the content of such sentences), make reference to utterances, or, more precisely, the 'that' in the noun clause is a demonstrative which refers to the utterance of the rest of the clause. Davidson argued that, if what followed the word 'that' referred instead to a sentence, then the replacement of the embedded sentential construction, which is understood to be both the (unstated) quotation of the embedded sentence and also the presumed sentential referent of the demonstrative, by a description of the sentence in terms of its syntax should not affect the sense of the resulting complex sentence. In other words, if we were to replace the embedded sentence "the earth moves" in (7) by a description of it in terms of the arrangement of its component symbols to obtain

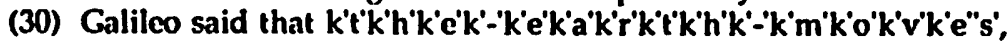
where ' $k$ ' is 'the concatenation of ... with ...., then the sense of (30) should not differ from that of (7). Since in general, and in this case in particular, the resulting sentence, if it indeed has any meaning at all, does not, of course, have a meaning that resembles anything like that of the original, the supposition that the referent of the two components of the 'that'-clause is the embedded sentence must be false. Although this line of reasoning correctly shows that the embedded sentence cannot be functioning as an appositive, this argument, I believe, misses the mark. The word 'that' in (7) functions similar to a name forming operator on the sentence "the earth moves" to form a name-like expression (or a 'quasiname') that refers to the sentence "the carth moves"; hence, the substitution of the syntactical description of the sentence for the actual syntactical construction in the name-like expression of the sentence produces what appears to be (and is) not a complex sentence but a meaningless string of symbols. Davidson has mistakenly attributed to the view that in sentences such as (7) reference is being made to a sentence, and not an ulterance, that it entails taking the embedded sentence as a sentence that is a name for itself.

Davidson's argument can be resuscitated, however, if it is claimed that a sentence with a completely different sense, if any sense at all, results from (7) upon the substitution of the syntactical description of the sentence for the entire 'that'-clause. However, the removal of the word 'that' in (7) changes the nature of the relationship between the subject and the embedded sentence. In other words, the word 'that' in the proposing phrase 'said that' both sets up a context in which the sentence following 'that' is referred to and figures in the specification of the relation referred to by the predicate expression. Yet, it may still be claimed that the substitution of the syntactical description of the quoted sentence in (6) for 
the entire expression composed of that sentence together with its quotation marks yields the same problematic sentence as was generated from (7). Does not this indicate that a sentence such as (6) is about a relation between a person and an utterance? And if this is the case, then, since a saying and a saying that are conceptually very similar, must a sentence such as (7) also be understood as expressing a relation between a person and an utterance? Negative answers can be given to both of these questions. If it were true that a sentence such as (6) expressed a relation between a person and an utterance, with the quotation being a name of the utterance, then the substitution in (6) of a description of a particular acoustical event constituting an utterance of the quoted sentence for the quoted sentence with its quotation marks should not alter the meaning of the original sentence. This, of course, is false, so by a counter-argument the construal of such a sentence as likewise involving a reference to an utterance must also be misguided.

The quoted sentence in (6) occurs in a context in which that sentence is being referred to, and the quotation marks serve both to signal that context and to form a name-like expression for the quoted sentence. This quasiname is a construction that occupies the grammatical position of a name but does not function like an ordinary name. Whereas there is no logical connection between a logically proper name and the object that the name refers to, the quasiname, whose construction constitutes a separate logical category, refers to a sentence in virtue of the connection in meaning and syntactical expression between the sentence and any complex sentence that contains the same quasiname. The reference to an object made by an ordinary singular term is what I call 'objectual reference', while the reference to a sentence made by such a quasiname is what I call 'substitutional reference'. A sentence containing the quasiname, which is a sentence of the metalanguage, does not treat the sentence referred to, which is a sentence of the object language, as an object since there are inferential links between the corresponding metalanguage and object language sentences. Both (6) and (7) contain quasinames of the sentence "The earth moves", and this common feature explains in part the similarity between these two sentences. The reason that substitution of a syntactical description for the embedded sentence does not preserve the sense of the original sentence is the same in the case of (6) as in the case of (7): when the embedded sentence (with or without its quotation marks or the word 'that') is removed, the quasiname in the original sentence is obliterated and meaning thus is not preserved.

The relation asserted to hold between Calileo and the sentence "The earth moves" is, however, not the same. The 'saying' relation mentioned in (6) would hold between Calileo and "The earth moves" in virtue of the former having made an utterance of a type that represented a verbal expression of the latter. The 'saying that' relation mentioned in (7) holds between Galileo and "The earth moves" in virtue of the former having 
made an utterance of a type that represented a verbal (or perhaps a written) expression of a sentence synonymous with the latter. 16 This explains why there is a conceptual similarity between the two kinds of sentences and accounts for why (6) is false and (7) is true. The 'saying' relation holds between a person who makes an utterance and a sentence with a certain syntactical structure revealed by the utterance. The 'saying that' relation holds between a person who makes an utterance and a sentence with a' certain semantical content that expresses what was meant by the utterance in its context. Since the syntactical features of the sentence referred to in a 'saying' relation are important while the semantical features of the sentence referred to in a 'saying that' relation are important, this is why (8) does not entail (9) and why (10) and (11) are mutually entailing. The reason why it is that (6) may entail (7) but (7) does not entail (6) is more complicated, but the above considerations constitute part of the explanation. The asymmetry in the inferential relations between these kinds of sentences is due to the nature of the conceptual connection between the 'saying' relation and the 'saying that' relation. However, when the speaker's utterance does not have its standard illocutionary force, the impermissibility of inferring a sentence such as (7) from its corresponding sentence of the form of (6) is due either to the objectual reference to the embedded sentence in the latter or to the consequent non-standard meaning of the complex sentence of the former.

Relations that can hold among sentences or between objects and sentences (such as the relations introduced by proposing phrases) do so obtain in virtue of the syntax or the semantics, or a combination of both, of the sentences substitutionally referred to, and, in spite of the fact that only one of these features of the embedded sentence may be relevant to particular relations, where substitutional reference to a sentence is made by an expression that expression does not make objectual reference to either the syntax or the semantics of the sentence. Although sometimes what may appear to be a case of substitutional reference is actually a case of objectual reference to an utterance, an inscription, a meaning, or a 'proposition', substitutional reference is not eliminable in favor of objectual reference. Sentences can be characterized in terms of their syntax or their semantics, but neither one of these in isolation suffices to individuate sentences. We quite often use the words 'sentence', 'string', or 'ulterance' when speaking of the syntax of the sentence and use the words 'meaning', 'proposition', or 'assertion' when speaking of the semantics of the sentence. The words 'statement' or 'sentence' is sometimes used when

16 The 'saying that' relation thus depends for its intelligibility on the synonymy relation. I will not endeavor to give an account here of synonymy. The non-logical nature of this relation affects the non-logical character of the 'saying that' relation, but such considerations do not affect the logical form of sentences containing the proposing phrase 'said that'. 
we wish to refer to a sentence neither solely in terms of its syntax nor solely in terms of its semantics. This latter is the manner in which I am using the ivord 'sentence'. When a sentence is referred to substitutionally, the particular object that is that sentence is never at issue. Instead, the (logically) complex sentence containing an expression that substitutionally refers is itself connected both syntactically and semantically with its embedded sentence (i.e., the sentence substitutionally referred to contributes both to the syntax and to the semantics of the complex sentence). Even though the sentence referred to appears syntactically within an expression that occupies the place of a name, that sentence is no more being treated as an object as is, for example, a conjunct in a conjunction.

However, since a quasiname of the kind under consideration does have a referring role, quantification over the position occupied by the quasiname is legitimate, as long as the quasiname is not itself a part of a longer quasiname. When a quasiname or a proper name occurs within a quasiname, they occur within an opaque context in which they, in effect, maintain their ordinary referents but their referring role is less direct, with the former having what I call 'secondary substitutional reference' and the latter having what I call 'secondary objectual reference'. Quantification over any position occupied by an expression having a secondary referring role is illegitimate since such quantification is into an opaque context. Quantification is meaningful only when it is over a position occupied by an expression having a direct, or 'primary', referring role since only in such contexts are expressions purely extensional. Quantification over the position occupied by a term having primary objectual reference must be understood objectually, whereas quantification over the position occupied by a quasiname having primary substitutional reference must be understood substitutionally. Since proposing phrases can be treated as introducing relations between persons and sentences, the above considerations account for both the inadmissibility of quantifying into the contexts within the embedded sentence and the admissibility of quantifying over the position occupied by the entire embedded sentence. Thus, the inference from (1) to (2) is justified, and the inference from (12) to (13) is not justified. Because sentences within the quasinames retain their integrity by not being assimilated into complex predicate expressions and preserve their meaning while still remaining components of complex sentences constructed from proposing phrases, embedded sentences can play a role, in certain circumstances, in deductions involving complex sentences about the propositional attitudes. 


\section{REFERENCES}

Davidson, Donald, "On Saying That", reprinted in The Philosophy of Language, ed. A.P. Martinich, 1985, Oxford University Press, pp. 339348.

Davidson, Donald, "Thought and Talk," Inquiries into Truth and Interpretation, 1984, Oxford: Clarendon Press, pp. 155-170.

Fodor, Jerry, "Propositional Attitudes", Monist, 61, no. 4 (October 1978), pp. 501-523.

Frege, Gottlob, "On Sense and Reference", reprinted in Translations from the Philosophical Writings of Goltlob Frege, eds. Peter Geach and Max Black, 1952, Oxford: Basil Blackwell, pp. 56-78.

Kaplan, David, "Quantifying In", reprinted in The Philosophy of Language, ed. A.P. Martinich, 1985, Oxford University Press, pp. 349-370.

Quine, W. V., "Quantifiers and Propositional Attitudes", reprinted in The Philosophy of Langunge, cd. A.l'. Martinich, 1985, Oxford University Press, pp. 332-338.

Quine, W.V., Word and Object, 1960, Cambridge, Massachusetts: M.I.T. Press, pp. 211-216.

Quine, W. V., Philosophy of Logic , second edition, 1970, Cambridge, Mass.:Harvard University Press, pp.15-34.

Russell, Bertrand, "On Denoting", reprinted in Contemporary Readings in Logical Theory, eds. Irving Copi and James Gould, 1967, New York: Macmillan, pp. 93-105.

Tarski, Alfred, "The Semantic Conception of Truth and the Foundations of Semantics", reprinted in The Philosophy of Language, ed. A.P. Martinich, 1985, Oxford University Press, pp. 48-71. 